\title{
Adrenal Ganglioneuroma: Presentation, Radiology, Histopathology, and Management
}

\author{
Liam A McMorrow ${ }^{1}$, Jessika Voll ${ }^{2}$, Andrew Elsaify ${ }^{3}$, Wael Elsaify ${ }^{4}$
}

\begin{abstract}
Aim and objective: Ganglioneuromas (GNs) are rare, benign tumors arising at peripheral autonomic ganglion sites. Their diagnosis is challenging and typically reliant on a histopathological diagnosis. We present two case studies of adrenal GN managed within our department alongside a review of the literature concerning the diagnosis and management of these unusual entities.

Materials and methods: We documented the presentation, investigation, and management of two patients with adrenal GN. Additionally, we conducted a literature review based on searching PubMed utilizing the term "adrenal ganglioneuroma".

Results: Both patients presented with an incidental indeterminate adrenal mass on imaging and underwent further laboratory investigations, abdominal ultrasound, computed tomography, and magnetic resonance imaging. In both cases, laparoscopic adrenalectomy was performed with specimens demonstrating adrenal GN on histopathological analysis. Our literature review further demonstrated the infrequency of these lesions and the difficulty involved in their diagnosis.

Conclusion: Ganglioneuroma occurs rarely in the adrenal gland usually without preceding clinical symptoms. Diagnosis is challenging and care must be taken to exclude malignant neuroblastoma and ganglioblastoma.

Keywords: Adrenal tumor, Adrenalectomy, Ganglioneuroma.

World Journal of Endocrine Surgery (2021): 10.5005/jp-journals-10002-1317
\end{abstract}

\section{INTRODUCTION}

Ganglioneuromas (GNs) are rare, benign tumors arising from neural crest cells at peripheral autonomic ganglion sites. Ganglioneuromas are typically found in the paravertebral sympathetic chains of the posterior mediastinum or retroperitoneum ( $79 \%$ of cases $\left.{ }^{1}\right)$. Twentyone percent are found in the adrenal gland with the remainder in the neck. ${ }^{1}$ The true incidence of adrenal GN is unknown and available literature is limited to the few available case reports. Current literature demonstrates increasing prevalence with age with diagnosis uncommon below the age of $30 .^{2}$ Adrenal GNs are rarely symptomatic and the majority of these tumors are detected incidentally. ${ }^{3}$ Although not without exception, adrenal GNs are typically non-functioning. ${ }^{4}$ An absence of laboratory detected abnormalities in conjunction with varying radiological findings adds to the difficulty in detecting and diagnosing GN. Currently, histopathological examination of tissue is required for diagnosis. We present two patients with adrenal GN and discuss current literature regarding its presentation, investigation, and management.

\section{Case Descriptions}

\section{Case 1}

A 46-year-old woman was investigated for left iliac fossa pain. Physical examination, observations, and routine laboratory tests were normal. Abdominal ultrasound was requested which demonstrated a large echopoor mass with echogenic foci measuring $8 \times 4.3 \mathrm{~cm}$ in size. Endocrine investigations including ACTH, DHEA, aldosterone, renin, plasma metadrenaline, androgen hormones, 24-hour urine metadrenaline, 24-hour normetadrenaline, and tumor markers (AFP, CA125, CEA, CA19-9) were normal. Abdominal CT demonstrated a well-defined lesion containing foci of calcification at LUQ lying between the stomach and left kidney measuring $77 \times 58 \mathrm{~mm}$ in the coronal plane. The

\footnotetext{
${ }^{1-4}$ Department of Breast and Endocrine Surgery, James Cook University Hospital, Middlesbrough, North Yorkshire, UK

Corresponding Author: Liam A McMorrow, Department of Breast and Endocrine Surgery, James Cook University Hospital, Middlesbrough, North Yorkshire, UK, e-mail: liammcmorrow@doctors.org.uk

How to cite this article: McMorrow LA, Voll J, Elsaify A, et al. Adrenal Ganglioneuroma: Presentation, Radiology, Histopathology, and Management. World J Endoc Surg 2021;13(1):28-31.

Source of support: Nil

Conflict of interest: None
}

lesion had an average density of $32 \mathrm{HU}$ on portal venous phase imaging (Fig. 1).

Magnetic resonance imaging demonstrated a $7.5 \times 7.5 \times 5.5$ $\mathrm{cm}$ mass arising from the left adrenal. The walls of this mass were well defined with non-invasive contact with the left spleen and kidney and were without cystic changes or hemorrhage. There was no macroscopic or intracellular lipid seen in the adrenal lesion. Contrast demonstrated delayed and progressive enhancement. Out of phase images did not show any significant signal dropout (Fig. 2).

Due to the size of the tumor and uncertain diagnosis, laparoscopic adrenalectomy was performed. Macroscopic examination revealed a left adrenal mass measuring $100 \times 70$ $\times 30 \mathrm{~mm}$. The cut surface of the lesion appeared whitish and lobulated. Histological examination revealed sheets of lesional spindle cells with intervening aggregates of ganglion cells. The intervening stroma showed foci of calcification and thick-walled blood vessels. There was no significant atypia, mitosis, or necrosis present. The lesional spindle cells expressed positivity for NFP, S100, synaptophysin (patchy), and GFAP (patchy) (Fig. 3). 

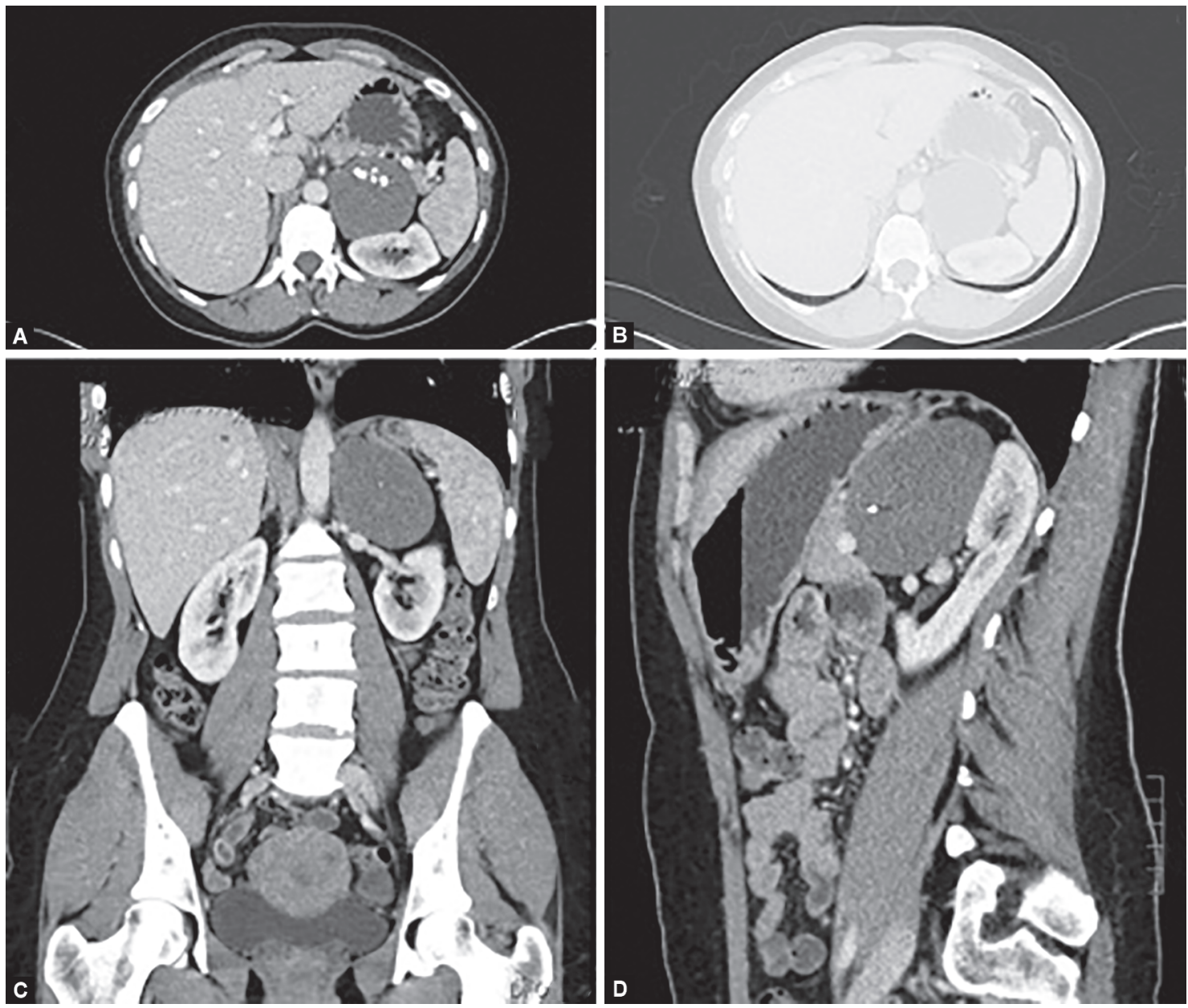

Figs 1 A to D: Abdominal CT demonstrating LUQ lesion $(77 \times 58 \mathrm{~mm})$ with an average density of $32 \mathrm{HU}$ on venous phase imaging

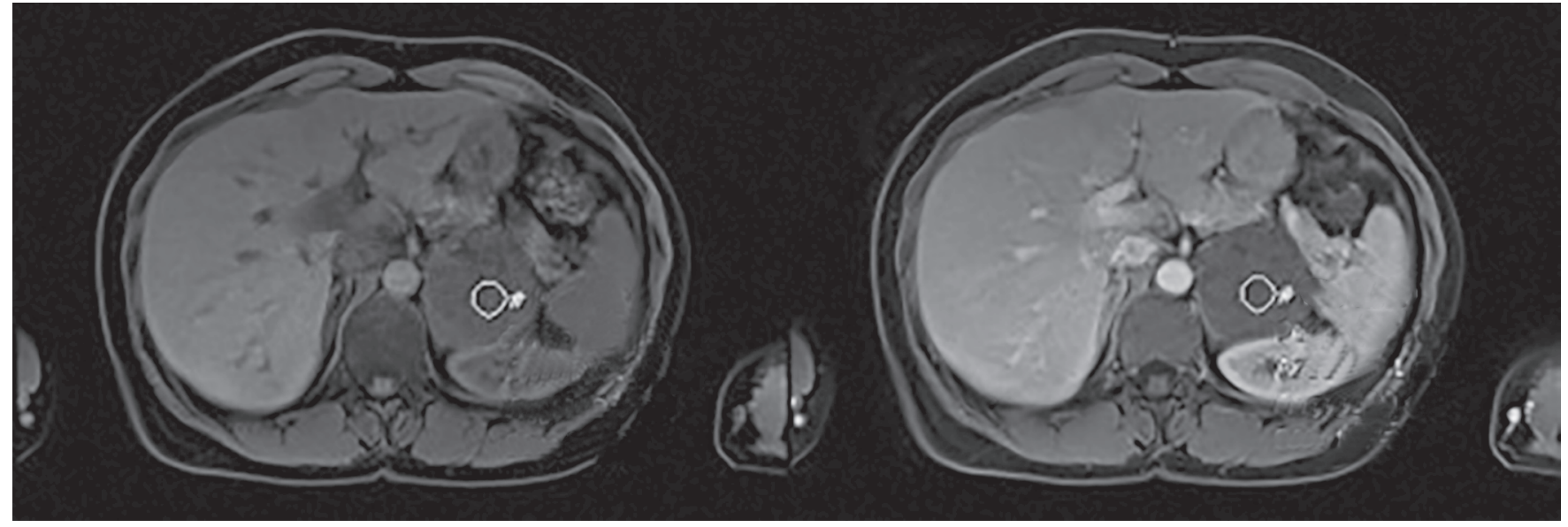

Fig. 2: T2-weighted axial magnetic resonance imaging demonstrating a heterogeneous left adrenal mass measuring $75 \times 75 \times 75 \mathrm{~mm}$ annotated to demonstrate delayed and progressive enhancement

They were negative for chromogranin, CD 99, CD 45, AE1/AE3, desmin, EMA, myogenin, HMB45, and WT1. The final histological diagnosis was that of GN. The patient recovered well from surgery without complication.

\section{Case 2}

A 51-year-old man was investigated for hematuria with a CT urogram which produced the incidental finding of a $5 \mathrm{~cm}$ right adrenal mass. Physical examination was without positive findings. 

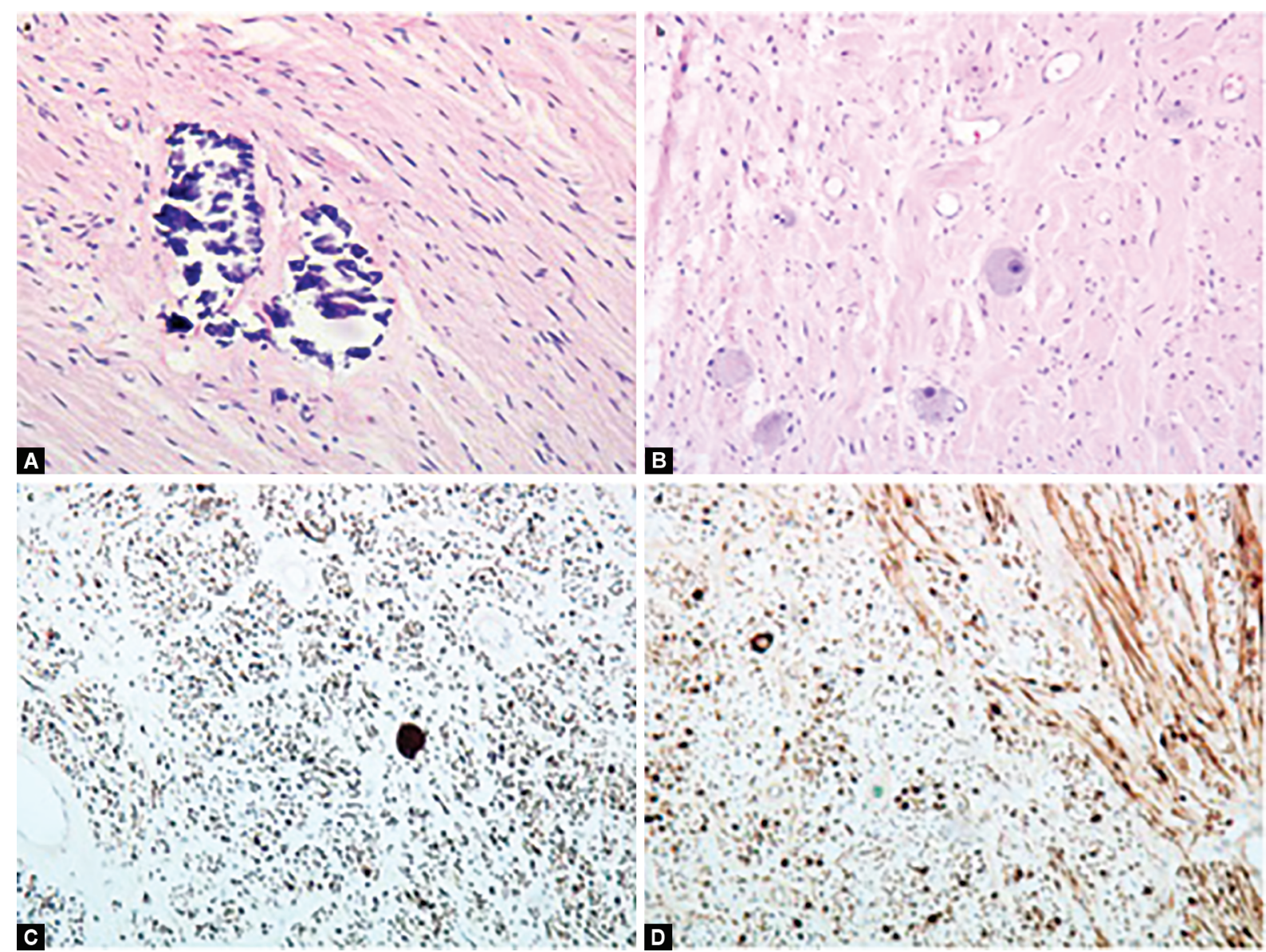

Figs 3 A to D: (A) The tumor in Case 1 showing schwann cells, spindle cells, nerve fibers and focal calcification; (B) Schwann cells, spindle cells, nerve fibres mixed with mature ganglion cells; (C) Positive staining for S100; (D) Positive staining for neurofilament protein (NFP)
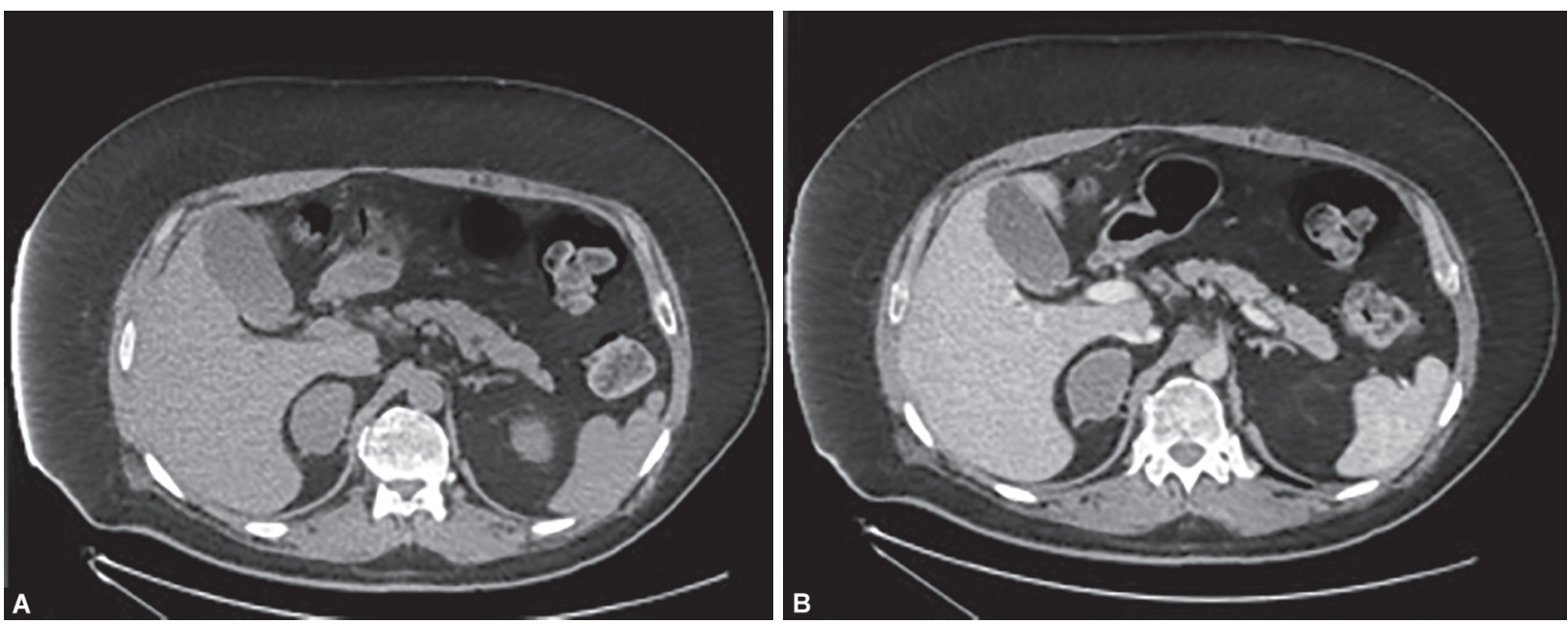

Figs 4A and B: Axial CT images from case 2 (A) pre- and (B) post-contrast administration (30.9 and 37.5 HU, respectively) demonstrating no significant enhancement

Past medical history was significant for non-insulin-dependent diabetes mellitus and paraplegia secondary to previous cervical spinal injury. The mass was non-functioning with normal routine blood tests and endocrine investigations including ACTH, DHEA, aldosterone, renin, plasma metadrenaline, and androgen hormones.
The CT urogram performed for hematuria had reported an indeterminate $5 \times 3.2 \mathrm{~cm}$ mass in the body of the right adrenal gland (Fig. 4). Contrast CT did not demonstrate significant enhancement of the lesion (Fig. 4). No cause for the hematuria was identified. Laparoscopic right adrenalectomy was performed and the patient recovered well from this without complication (Fig. 4). 
Macroscopic examination revealed a yellow-brown tumor within the right adrenal gland. The cut surface of the lesion revealed a white whorled appearance with a yellow rim and measuring 50 $\mathrm{mm}$ in its maximum diameter. Histological examination revealed bland spindle cells in trabeculae, fat and mature ganglion cells. There was no necrosis or cytological atypia. The lesional spindle cells expressed positivity for $\mathrm{S} 100$ and negativity for desmin. The histological diagnosis was that of benign GN.

\section{Discussion}

Ganglioneuromas are rare benign tumors of the sympathetic nervous system. They join the family of peripheral neuroblastic tumors which include malignant neuroblastoma and ganglioblastoma. Although GN is a benign tumor, there are case reports of GN presenting with differentiated metastases. ${ }^{5,6}$ This phenomenon is explained through spontaneous cytomaturation of neuroblastoma. Neuroblastoma is noted for its ability to spontaneously regress or mature to a benign form.

In addition to the neuroblastic tumor family, the differential diagnosis for adrenal GN includes the adrenal solid masses; pheochromocytoma, adenoma, and carcinomas.

While case reports describing endocrinologically active GN's can be found, the majority of adrenal GNs are hormonally inactive and without clinical symptoms. ${ }^{4}$ Elevated urinary and plasma catecholamines are more typical off and suggestive of neuroblastoma as opposed to the benign GN. With the exception of a reported association with MEN $2 b^{7}$ and neurofibromatosis type $\mathrm{I}^{8}$ there are no known risk factors.

Although usually clinically silent, patients presenting with symptoms typically present with non-specific abdominal symptoms.

Radiological diagnosis is challenging and studies report high rates of misdiagnosis. Adrenal GN is typically described as a wellcircumscribed tumor with a lobular shape and low attenuation. Radiological features on CT/MRI may be similar to that of adrenocortical cancer and pheochromocytoma. ${ }^{1}$

Ultrasound findings are typically that of a well-defined, hypoechogenic homogeneous mass. MRI and CT currently represent the method of choice for imaging ganglioblastomas.

It is not possible to fully differentiate between the neuroblastic tumors radiologically as they typically involve similar features. ${ }^{9}$ As stated earlier, while the presence of metastases would not be seen with GN, there are rare exceptions to this.

Neuroblastic tumors typically appear on CT as wellcircumscribed smooth masses ranging from round to lobular in shape and containing calcifications. They are hypoattenuating to muscle and may show delayed heterogeneous contrast uptake typically proportionate to the presence of myxoid stroma. ${ }^{10}$ GN's typically surround major blood vessels without invasion or compression. ${ }^{11}$ Utilizing MRI GNs are homogeneous with low/ intermediate signal intensity on T1 and heterogeneous with intermediate/high signal intensity on T2. There is typically no change in signal intensity on chemical shift imaging. Apparent diffusion coefficient values are typically higher in GN than in neuroblastoma. ${ }^{11}$ 131-lodine-metaiodobenzylguanidine (131 I-MIBG) scan can be useful in functioning masse ${ }^{12}$ but is typically more useful in cases of neuroblastoma or ganglioblastoma.

Diagnosis remains histological. Ganglioneuroma is welldifferentiated and consists microscopically of ganglion cells, Schwann cells, and fibrous tissue. Grossly, these lesions are typically large and encapsulated with a homogeneous gray/white appearance. Ganglioneuromas are characterized by reactivity for S-100, vimentin, synaptophysin, and neuronal markers. ${ }^{13}$

To conclude, adrenal $\mathrm{GN}$ is a rare and challenging diagnosis usually dependent on histopathological findings. These rare case reports demonstrate adrenal GN presenting incidentally and as a cause of non-specific abdominal symptoms and the extent of investigations required in its diagnosis and treatment. Care must be taken to exclude the malignant neuroblastoma and ganglioblastoma.

\section{References}

1. Majbar A, Al E. Imaging of adrenal ganglioneuroma: a case report. BMC Res Notes 2014(7):791. DOI: 10.1186/1756-0500-7-791.

2. Barzon L, Sonino N, Fallo F, et al. Prevalence and natural history of adrenal incidentalomas. Eur J Endocrinol 2003;149(4):273-285. DOI: 10.1530/eje.0.1490273.

3. Zhou Y, Liang Q, Ou W-T, et al. Laparoscopic resection of primary adrenal ganglioneuroma: a case report and review of the literature. Oncol Lett. 2015;9(5):2167-2170. DOI: 10.3892/ol.2015.3021.

4. Lucas K, Gula MJ, Knisely AS, et al. Catecholamine metabolites in ganglioneuroma. Med Pediatr Oncol 1994;22(4):240-243. DOI: $10.1002 / m p o .2950220405$.

5. Jung HR, Kang K-J, Kwon JH, et al. Adrenal ganglioneuroma with hepatic metastasis. J Korean Surg Soc 2011;80(4):297. DOI: 10.4174/ jkss.2011.80.4.297.

6. Garvin JH, Lack EE, Berenberg W, et al. Ganglioneuroma presenting with differentiated skeletal metastases. Report of a case. Cancer 1984;54(2):357-360. DOI: 10.1002/1097-0142(19840715)54:2<357::AIDCNCR2820540230>3.0.CO;2-4.

7. Lora MS, Waguespack SG, Moley JF, et al. Adrenal ganglioneuromas in children with multiple endocrine neoplasia type 2: a report of two cases. J Clin Endocrinol Metab 2005;90(7):4383-4387. DOI: 10.1210/ jc.2004-2526.

8. Geraci AP, de Csepel J, Shlasko E, et al. Ganglioneuroblastoma and ganglioneuroma in association with neurofibromatosis type I: report of three cases. J Child Neurol 1998;13(7):356-358. DOI: 10.1177/088307389801300712. http://www.ncbi.nlm.nih.gov/ pubmed/9701489. Accessed November 12, 2016.

9. Lonergan GJ, Schwab CM, Suarez ES, et al. Neuroblastoma, ganglioneuroblastoma, and ganglioneuroma: radiologic-pathologic correlation. Radiographics 2002;22(4):911-934. DOI: 10.1148/ rg. 232025146.

10. Zhang $\mathrm{Y}$, Nishimura $\mathrm{H}$, Kato $\mathrm{S}$, et al. MRI of ganglioneuroma: histologic correlation study. J Comput Assist Tomogr. 25(4):617-623. DOI: 10.1097/00004728-200107000-00018. http://www.ncbi.nlm.nih.gov/ pubmed/11473195. Accessed November 12, 2016.

11. Ichikawa T, Ohtomo K, Araki T, et al. Ganglioneuroma: computed tomography and magnetic resonance features. $\mathrm{Br} J$ Radiol 1996;69(818):114-121. DOI: 10.1259/0007-1285-69-818-114.

12. Geoerger B, Hero B, Harms D, et al. Metabolic activity and clinical features of primary ganglioneuromas. 2001(10):1905-1913. DOI: 10.1002/1097-0142(20010515)91:103.0.co;2-4.

13. WHO Classification of Tumours of the Central Nervous System. World Health Organization; 2007. 